\title{
Hybrid Collocated PD with Non-collocated PID for Sway Control of a Lab-scaled Rotary Crane
}

\author{
M.A. Ahmad, M.S. Ramli, M.A. Zawawi, R.M.T. Raja Ismail \\ Faculty of Electrical and Electronics Engineering \\ Universiti Malaysia Pahang \\ Lebuhraya Tun Razak, 26300, Kuantan, Pahang, Malaysia
}

\begin{abstract}
This paper presents investigations into the development of hybrid control schemes for sway suppression and rotational angle tracking of a rotary crane system. A lab-scaled rotary crane is considered and the dynamic model of the system is derived using the Euler-lagrange formulation. To study the effectiveness of the controllers, initially a collocated proportionalderivative (PD) controller is developed for control of rotary motion. This is then extended to incorporate a noncollocated PID controller for control of sway angle of the pendulum. Implementation results of the response of the rotary crane system with the controllers are presented in time and frequency domains. The performances of the control schemes are assessed in terms of level of sway reduction, rotational angle tracking capability and time response specifications. Finally, a comparative assessment of the control techniques is presented and discussed.
\end{abstract}

Index Terms - Rotary crane, sway control, collocated PD, non-collocated PID controller.

\section{INTRODUCTION}

The main purpose of controlling a crane system is transporting the load as fast as possible without causing any excessive sway at the final position. However, most of the common crane results in a sway motion when payload is suddenly stopped after a fast motion [1]. The sway motion can be reduced but will be time consuming. Moreover, the crane system needs a skillful operator to control manually based on his or her experiences to stop the swing immediately at the right position. The failure of controlling crane also might cause accident and may harm people and the surrounding.

Although cranes are very important systems for handling heavy goods, automatic cranes are comparatively rare in industrial practice [2], because of high investment costs. The need for faster cargo handling requires control of the crane motion so that its dynamic performance is optimized. Specifically, the control of overhead crane systems aims to achieve both position regulation and anti-swing control. Several authors have looked at this including [3], time-optimal control was considered using boundary conditions, an idea which was further developed in [4] and [5]. Unfortunately, to increase robustness, some time optimization requirements, like zero angular velocity at the target point [6], have to be given 978-1-4244-5046-6/10/\$26.00 @2010 IEEE up. Gain scheduling has been proposed as a practicable method [7] to increase tracking accuracy, while observerbased feedback control was presented in [2]. Many attempts, such as planar operation [7] and assuming the absence of friction [6], have been made to introduce simplified models for application of model-based control [2]. Thus, a self-tuning controller with a multilayer perceptron model for an overhead crane system was proposed [8] while in reference $[9,10]$, the controller consists of an active sway control of a gantry crane system with disturbance effect.

This paper is organized as follows: Section II describes the lab-scaled rotary crane system. Section III discusses a brief description on the mathematical modelling of rotary crane system. Section IV is devoted to develop a rotational angle tracking and sway reduction control schemes for rotary crane system. Implementation results are shown in section $\mathrm{V}$ and conclusions are drawn in section VI.

\section{THE RotARy CRANE SYSTEM}

The 2-DOF rotary crane system with its payload considered in this work is shown in Figure 1, where $\theta$ and $\alpha$ denote the horizontal angle of the arm and the sway angle of the pendulum, respectively, $r$ and $L$ is the length of arm and pendulum. In this simulation, the pendulum and payload can be considered as point masses. The lab-scaled rotary crane system is shown in Figure 2.

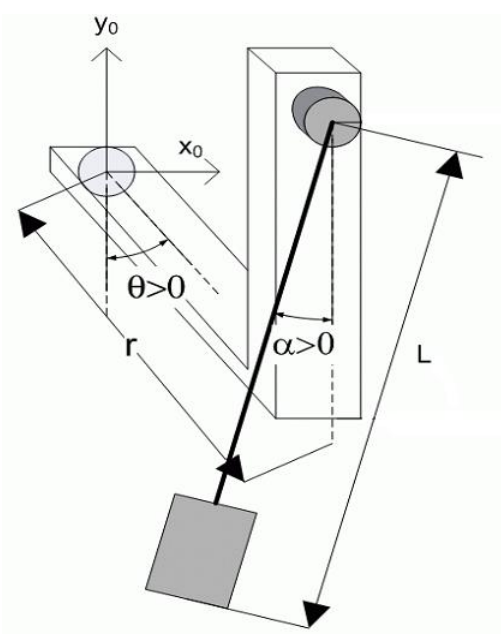

Figure 1: Description of the rotary crane system.

ICIEA 2010 


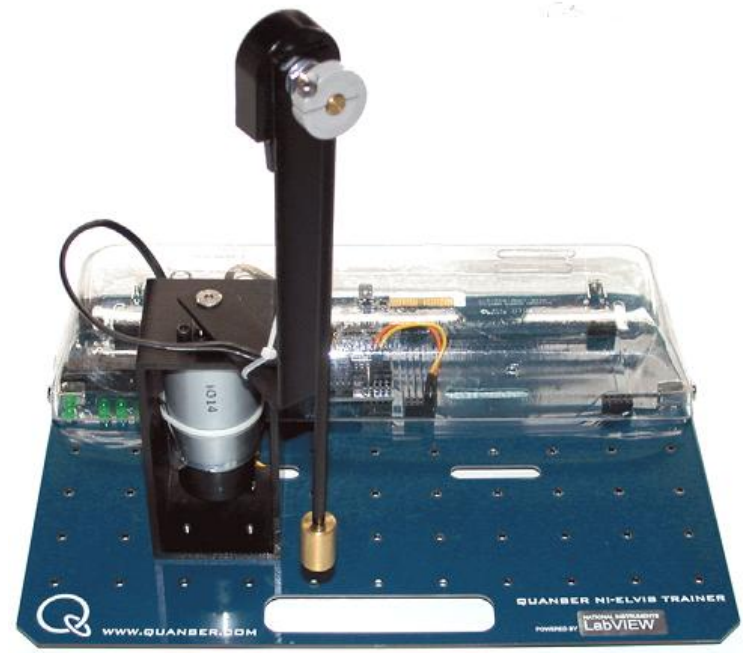

Figure 2: Lab-scaled rotary crane system.

\section{DYNAMIC MODELING OF THE RotARy CRANE}

This section provides a brief description on the modeling of the rotary crane system, as a basis of a simulation environment for development and assessment of the hybrid control techniques. The Euler-Lagrange formulation is considered in characterizing the dynamic behavior of the crane system incorporating payload.

Considering the motion of the rotary crane on a horizontal plane, the kinetic energy of the system can thus be formulated as [11]

$$
\begin{aligned}
T= & \frac{1}{2} J_{a} \dot{\theta}^{2}+\frac{1}{2} m(L \cos \alpha(\dot{\alpha})+r \dot{\theta})^{2} \\
& +\frac{1}{2} m(L \sin \alpha(\dot{\alpha}))^{2}+\frac{1}{2} J_{p} \dot{\alpha}^{2}
\end{aligned}
$$

The potential energy of the beam can be formulated as

$$
U=m g l(1-\cos \alpha)
$$

To obtain a closed-form dynamic model of the rotary crane, the energy expressions in (1) and (2) are used to formulate the Lagrangian $L=T-U$. Let the generalized torques corresponding to the generalized state variables $\bar{q}=\{\theta, \alpha\}$ be $\bar{\tau}=\{\tau, 0\}$. Using Lagrangian's equation

$$
\begin{aligned}
& \frac{d}{d t}\left(\frac{\partial L}{\partial \dot{\theta}}\right)-\frac{\partial L}{\partial \theta}=\tau-B \dot{\theta} \\
& \frac{d}{d t}\left(\frac{\partial L}{\partial \dot{\alpha}}\right)-\frac{\partial L}{\partial \alpha}=0
\end{aligned}
$$

and linearizing $\alpha=0$, the equation of motion is obtained as below,

$$
\begin{aligned}
& \left(J_{a}+m r^{2}\right) \ddot{\theta}+m L r \ddot{\alpha}=\tau-B \dot{\theta} \\
& \frac{4}{3} m L^{2} \ddot{\alpha}+m L r \ddot{\theta}+m g L \alpha=0
\end{aligned}
$$

where $m$ is the centre of mass of pendulum and payload, payload, $B$ is the arm viscous damping, $J_{a}$ and $J_{p}$ denotes the moment of inertia of the arm and pendulum respectively and $g$ is the gravity effect.

In this study the values of the parameters are defined as $m=0.027 \mathrm{~kg}, J_{a}=1.23 \times 10^{-4} \mathrm{~kg}-\mathrm{m}^{2}, L=0.191 \mathrm{~m}, r=0.06668$, $B=0.001 \mathrm{Nm} / \mathrm{rad} / \mathrm{s}, g=9.81 \mathrm{~m}-\mathrm{s}^{-2}[10]$.

\section{CONTROLLER DESIGN}

In this section, control schemes for rotary motion control and sway suppression of a rotary crane are proposed. Initially, a collocated PD controller is designed. Then a non-collocated PID control is incorporated in the closed-loop system for control of sway of the system.

\section{A. Collocated PD controller}

A common strategy in the control of rotary crane systems involves the utilization of PD feedback of collocated sensor signals. In this work, such a strategy is adopted at this stage. A block diagram of the PD controller is shown in Figure 3, where $K_{p}$ and $K_{v}$ are proportional and derivative gains, respectively, $\theta$ and $\dot{\theta}$ represent hub angle and hub velocity, respectively, $R_{f}$ is the reference hub angle and $A_{c}$ is the gain of the motor amplifier. Here the motor/amplifier gain set is considered as a linear gain. To design the PD controller, a linear state-space model of the rotary crane was obtained by linearising the equations of motion of the system. The tracking performance of the collocated PD control applied to the rotary crane system was investigated based on the root locus analysis, from which $K_{p}, K_{v}$ and $A_{c}$ were deduced as $0.0323,0.0067$, and 1.969 respectively.

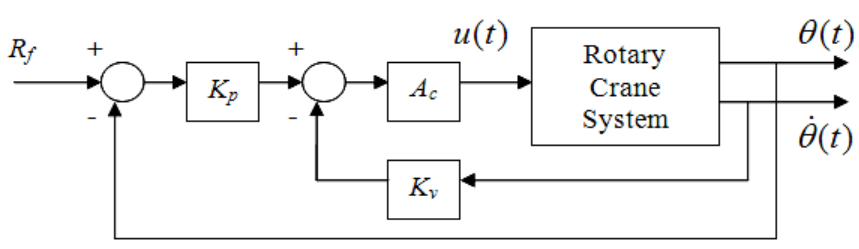

Figure 3: The collocated PD control structure.

\section{B. Collocated PD with non-collocated PID controller}

A combination of collocated PD and non-collocated PID control scheme for control of rotary motion and sway suppression of the system is presented in this section. The use of a non-collocated control system, where the sway of the 
rotary crane is controlled by measuring its angle, can be applied to improve the overall performance, as more reliable output measurement is obtained. The control structure comprises two feedback loops: (1) The hub angle as input to collocated PD for rotary motion control. (2) The sway angle as input to a separate non-collocated control law for sway control. These two loops are then summed together to give a torque input to the system. A block diagram of the control scheme is shown in Figure 4 where $\alpha(t)$ represents the sway angle of the pendulum. $r_{\alpha}$ represents sway angle reference input, which is set to zero as the control objective is to have zero sway during rotational movement of the crane.

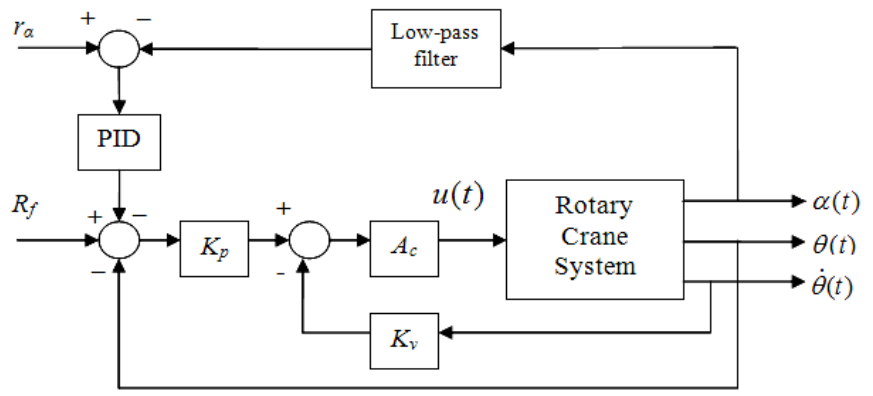

Figure 4: Collocated PD with non-collocated PID control structure.

For rotary motion control, the collocated PD control strategy developed in the previous section is adopted whereas for the sway control loop, the sway angle of pendulum feedback through a PID control scheme is utilised. The PID controller parameters were tuned using the Ziegler-Nichols method using a closed-loop technique, where the proportional gain $K_{p}$ was initially tuned and the integral gain $K_{i}$ and derivative gain $K_{d}$ were then calculated. Accordingly, the PID parameters $K_{p}, K_{i}$ and $K_{d}$ were deduced as 5.46, 14.89 and 0.05 respectively. To decouple the sway angle measurement from the rotary motion of the rotary crane, a second-order infinite impulse response (IIR) Butterworth Low-pass filter was utilised. In this investigation, a Low-pass filter with cutoff frequency of $15 \mathrm{~Hz}$ was designed.

\section{IMPLEMENTATION AND RESULTS}

In this section, the proposed control schemes are implemented and tested to the lab-scaled rotary crane environment and the corresponding results are presented. The rotary crane is required to follow a trajectory motion of 90 degree. System responses namely the hub angle of the arm and sway angle of the pendulum are observed. To investigate the sway of the system in the frequency domain, power spectral density (PSD) of the sway angle response is obtained. The performances of the control schemes are assessed in terms of sway suppression, input tracking and time response specifications. Finally, a comparative assessment of the performance of the control schemes is presented and discussed.
Figures 5-7 show the responses of the rotary crane to the reference input trajectory using collocated PD in time-domain and frequency domain (PSD). These results were considered as the system response under rotary motion control and will be used to evaluate the performance of the non-collocated PID control. The steady-state hub angle trajectory of 90 degree for the rotary crane was achieved within the rise and settling times and overshoot of $0.474 \mathrm{~s}, 0.713 \mathrm{~s}$ and $1.72 \%$ respectively. A noticeable amount of sway occurs during movement of the rotary crane. It is noted from the sway of the pendulum response that the sway of the system remain oscillates within 5 $\mathrm{s}$ with a maximum residual of \pm 14 degree. Moreover, from the PSD of the sway angle response the system are dominated by a single mode of sway, which are obtained as $0.9766 \mathrm{~Hz}$ with magnitude of $49.98 \mathrm{~dB}$.

The hub angle, sway angle and power spectral density responses of the rotary crane with PDPID control are shown in Figures 5-7 respectively. It is noted that the proposed control schemes are capable of reducing the system sway while maintaining the input tracking performance of the manipulator. Similar hub angle, sway angle and power spectral density of sway angle responses were observed as compared to the collocated PD contol. With PDPID, the steady-state hub angle trajectory of 90 degree for the rotary crane was achieved within the rise and settling times and overshoot of $0.628 \mathrm{~s}$, $1.384 \mathrm{~s}$ and $2.80 \%$ respectively. It is noted that the trajectory responses of PDPID is slower as compared to the case of PD control. However, a significant amount of sway reduction was demonstrated at the sway angle of the pendulum with PDPID. Moreover, from the sway angle response, the system sway settles within $1.5 \mathrm{~s}$ with a maximum residual of \pm 10 degree. The sway suppression is also evidenced from the PSD of the sway angle response where the magnitude of sway was reduced to $12.33 \mathrm{~dB}$ for the single mode of sway.

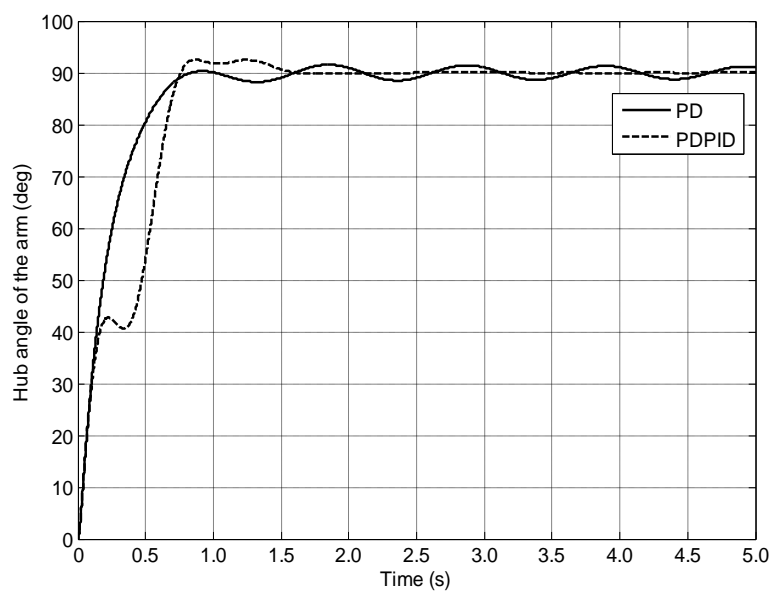

Figure 5: Hub angle response with PD and PDPID control. 


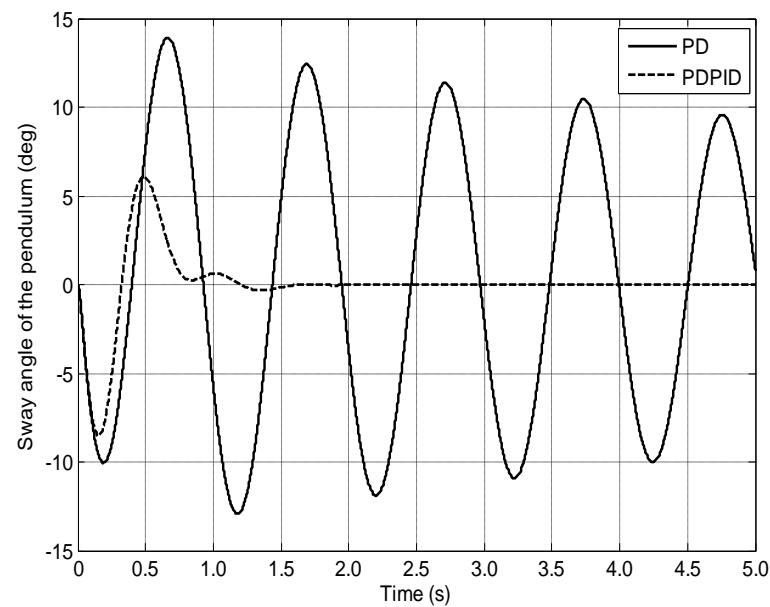

Figure 6: Sway angle response with PD and PDPID control.

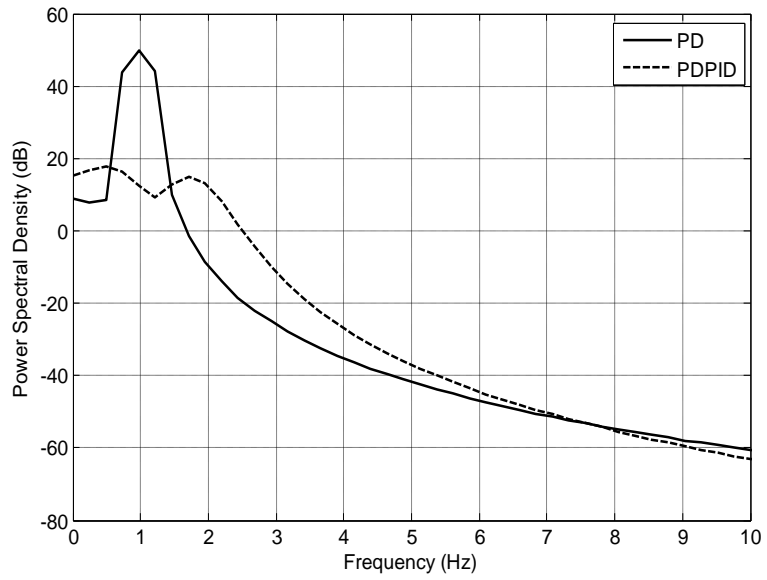

Figure 7: PSD response with PD and PDPID control.

Table 1 summarises the magnitude of sway of the pendulum and specifications of hub angle response for both control schemes. It is noted that high performance in the reduction of sway of the system is achieved using PDPID control. This is observed and compared to the collocated PD control at the single mode of sway. For comparative assessment, the level of sway reduction of the pendulum was obtained as $37.65 \mathrm{~dB}$ at the $0.9766 \mathrm{~Hz}$ sway frequency. Moreover, almost twofold improvement in the sway reduction was observed with PDPID as compared to the collocated PD. However, as demonstrated in the hub angle trajectory response, slightly slower response is obtained using collocated PD with non-collocated PID control as compared to the collocated PD. Comparisons of the specifications of the hub angle trajectory responses are summarised in Table 1 and further evidenced are shown in a bar graph in Figure 8. Besides, as demonstrated in the hub angle trajectory response with PDPID control, the minimum phase behaviour of the crane is unaffected. Nevertheless, the implementation of PDPID required a large amount of design effort in order to determine the best PID parameters. Note that a properly tuned PID could produce better results.

TABLE I

MAGNITUDE OF SWAY AND SPECIFICATIONS OF HUB ANGLE RESPONSE

\begin{tabular}{cccc}
\hline \hline \multicolumn{2}{c}{ Controller } & PD & PDPID \\
\hline \hline \multicolumn{2}{c}{ Magnitude of sway $(\mathrm{dB})$} & 49.98 & 12.33 \\
\hline \multirow{2}{*}{$\begin{array}{c}\text { Specifications } \\
\text { of hub angle } \\
\text { response }\end{array}$} & Settling time (s) & 0.713 & 1.384 \\
\hline \hline
\end{tabular}

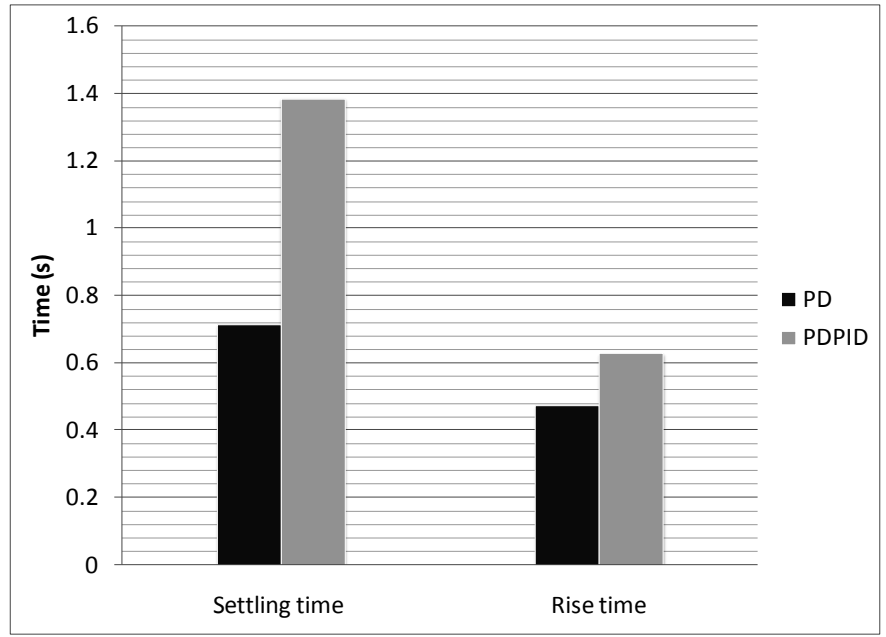

Figure 8: Rise and settling times of the hub angle response with PD and PDPID control.

\section{CONCLUSIONS}

The development of techniques for sway angle suppression and rotational angle tracking of a lab-scaled rotary crane has been presented. The control schemes have been developed based on collocated PD and collocated PD with non-collocated PID control. The performances of the control schemes have been evaluated in terms of sway suppression and input tracking capability at the resonance mode of the pendulum's sway. Acceptable performance in sway suppression and input tracking control has been achieved with proposed control strategies. A significant amount of sway reduction of the pendulum was demonstrated with PDPID control. However, in term of speed of responses, PDPID control results in a slower tracking response with more overshoot as compared to collocated PD. The work thus developed and reported in this paper forms the basis of design and development of hybrid control schemes for input tracking and sway suppression for others type of crane system.

\section{ACKNOWLEDGMENT}

This work was supported by Faculty of Electrical \& Electronics Engineering, Universiti Malaysia Pahang, 
especially Control \& Instrumentation (COINS) Research Group.

\section{REFERENCES}

[1] H.M., Omar, "Control of gantry and tower cranes", Ph.D. Thesis, M.S. Virginia Tech, 2003.

[2] O. Sawodny, H. Aschemann and S. Lahres, "An automated gantry crane as a large workspace robot", Control Engineering Practice, Vol. 10, No. 12, pp. 1323-1338, 2002.

[3] J.W. Beeston, "Closed-loop time optimatial control of a suspended payload - a design study", Proc. 4th IFAC World Congress, pp. 85-99, Warsaw Poland, 1969

[4] J.W. Auernig and H. Troger, "Time optimal control of overhead cranes with hoisting of the payload", Automatica, Vol. 23, No. 4, pp. 437-447, 1987.

[5] Y. Sakawa and Y. Shindo, "Optimal control of container cranes", Automatica, Vol. 18, No. 3, pp. 257-266, 1982.

[6] M.W. Noakes and J.F. Jansen, "Generalized input for damped-vibration control of suspended payloads", Journal of Robotics and Autonomous Systems, Vol. 10, No. 2, pp. 199-205, 1992.

[7] G. Corriga, A. Giua and G. Usai, "An implicit gain-scheduling controller for cranes", IEEE Trans. Control Systems Technology, Vol. 6, No. 1, pp. 15-20, 1998.

[8] J.A. Mendez, L. Acosta, L. Moreno, S. Torres and G.N. Marichal, "An application of a neural self-tuning controller to an overhead crane", Neural Computing and Applications, Vol. 8, No. 2, pp. 143-150, 1999.

[9] M.A. Ahmad, H. Ishak, A.N.K. Nasir and M.S. Ramli, "Comparison of Active Sway Control of a Gantry Crane System", Proceedings of $3^{\text {rd }}$ Asia International Conference on Modelling and Simulation, Bandung, 25-26 May 2009, pp. 431-435.

[10] M.A. Ahmad, A.N.K. Nasir, M.S. Najib, H. Ishak, "Anti-sway techniques in feedback control loop of a gantry crane system A comparative assessment of PD and PD-type fuzzy logic controller", Proceedings of The $4^{\text {th }}$ IEEE International Conference on Industrial Electronics and Applications, Xi'an, China, 25-27 May 2009, pp. 24832487.

[11] Quanser Student Handout, Rotary Pendulum Gantry Trainer. http://www.quanser.com. 